[12] L. Mirkin, H. Rotstein, and Z. J. Palmor, " $H^{2}$ and $H^{\infty}$ design of sampled-data systems using lifting-Part I: General framework and solutions," Faculty of Mechanical Engineering, Technion-Israel Institute of Technology, Tech. Rep. TME-450, Sept. 1997.

[13] _ " " $H^{2}$ and $H^{\infty}$ design of sampled-data systems using lifting-Part II: Properties of systems in the lifted domain," Faculty of Mechanical Engineering, Technion-Israel Institute of Technology, Tech. Rep. TME-451, Sept. 1997.

[14] L. Mirkin and Z. J. Palmor, "On the sampled-data $H^{\infty}$ filtering problem," Automatica, to be published.

[15] L. Mirkin and H. Rotstein, "On the characterization of sampled-data controllers in the lifted domain," Syst. Contr. Lett., vol. 29, no. 5, pp. 269-277, 1997.

[16] Y. Hayakawa, S. Hara, and Y. Yamamoto, " $H^{\infty}$ type problem for sampled-data control systems-A solution via minimum energy characterization," IEEE Trans. Automat. Contr., vol. 39, pp. 2278-2284, Nov. 1994.

\section{A Recursive Algorithm for ARMAX Model Identification in Closed Loop}

\author{
I. D. Landau and A. Karimi
}

\begin{abstract}
The joint problem of the recursive estimation of an optimal predictor for the closed-loop system and the unbiased parameter estimation of an ARMAX plant model in closed-loop operation is considered. A special reparameterized optimal predictor for the closed-loop is introduced. This allows a parameter estimation algorithm for the plant model to be derived which is globally asymptotically stable in a deterministic environment and gives asymptotically unbiased parameters estimates under richness conditions.
\end{abstract}

Index Terms-ARMAX model, closed-loop identification, output error, recursive algorithm.

\section{INTRODUCTION}

The plant model identification in closed-loop operation is an important practical issue. In many cases the plant can be operated in open loop with difficulties or a controller may already exist and it is not possible to open the loop. Furthermore, for controller maintenance it is convenient to reidentify the plant in closed-loop operation and retune the controller. A number of techniques for plant model identification in closed-loop have been developed and analyzed in detail [1]-[3].

The interest to the problem of closed-loop identification has been enhanced in the recent years in the context of iterative combination of the identification in closed-loop and robust control redesign [4]. In this context the objective is to identify a new plant model in closed-loop operation such that a better predictor for the closed-loop

Manuscript received September 23, 1997. Recommended by Associate Editor, J. C. Spall.

I. D. Landau is with the Laboratoire d'Automatique de Grenoble CNRSINPG-UJF, ENSIEG, 38402 Saint Martin d'Hères, France (e-mail: landau@lag.ensieg.inpg.fr).

A. Karimi is with the Electrical Engineering Department, Sharif University of Technology, Tehran, Iran.

Publisher Item Identifier S 0018-9286(99)02113-3. is obtained. While this idea was mentioned in [1], it has not been developed until recently.

Starting from the objective mentioned above, the plant model identification can be viewed as a model reference adaptive system (MRAS) identification problem where the reference model is the true closed-loop system and the adjustable system has the form of the design system. Alternatively, one can view this problem as the identification of the closed-loop with a reparameterized predictor in terms of the estimated plant model and the controller (which is known and constant).

The MRAS approach has led to an "output error" type closedloop recursive identification algorithm [5]. This algorithm requires a positive real condition to be satisfied in both the deterministic and the stochastic environment. This condition depends upon the parameters of the controller and the characteristic polynomial of the closed loop. Asymptotic unbiased parameter estimates are obtained if the noise disturbing the output of the plant is independent with respect to the external excitation.

In practice a very useful plant model for system identification is the ARMAX model for which a number of recursive algorithms for identification in open-loop are available (extended least squares, recursive maximum likelihood, output error with extended prediction model, $\cdots$ ).

The objective of this paper is to develop a recursive ARMAX model identification algorithm for plants operating in closed-loop such that:

- global asymptotic stability of the algorithm is assured for any initial parameter estimates, and initial error between the outputs of the two systems (in the absence of noise);

- an asymptotically optimal predictor of the true closed-loop system is obtained;

- asymptotically unbiased estimates of the plant model parameters are obtained under the richness conditions;

under the assumptions that: 1) the plant belongs to the model set; 2) the controller is stable, constant, and known; and 3) an external excitation is applied to the closed-loop system.

The algorithm is obtained by starting from an appropriate reparameterization of the optimal predictor for the closed loop. The objective of the algorithm is to asymptotically whiten the error between the two systems (i.e., the adjustable closed-loop system will become an asymptotically optimal predictor for the true closed-loop system). With respect to the algorithm given in [5] the resulting algorithm called X-CLOE (for extended closed-loop output error) does not require any positive real condition for global asymptotic stability in deterministic environment. For global convergence toward unbiased parameter estimates it requires a positive real condition which depends only on the noise model (like for the modified extended least squares (AML) [6] and the output error with extended prediction model [7], [8] used for ARMAX plant model identification in open-loop operation) and not upon the characteristics of the closed loop.

The paper will focus on the analysis of the algorithm both in deterministic and in stochastic environment. The simulation and the experimental evaluation of this algorithm are presented in [9]. For the case that the plant model is not in the model set an analysis of the bias distribution in the frequency domain can be carried out [10]. The paper is organized as follows. In Section II the recursive parameter estimation algorithm is derived. The stability analysis is presented in Section III. The convergence properties in the stochastic environment 


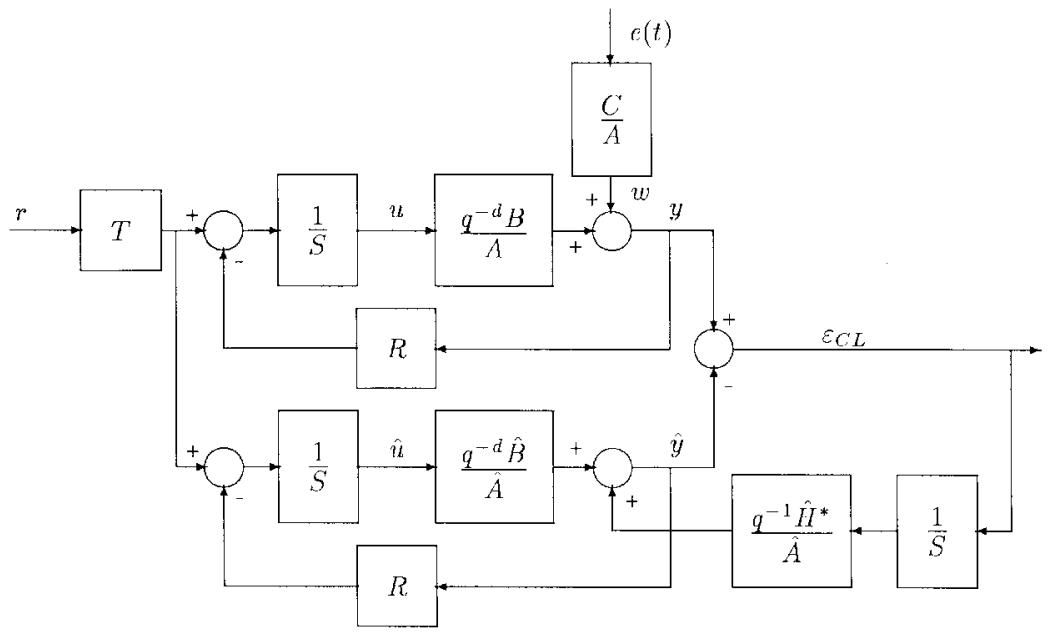

Fig. 1. X-CLOE identification scheme.

are examined in Section IV using martingale approach. Conclusions are given in Section V.

\section{THE ALgORITHM}

The objective is to estimate the parameters of the plant model defined by the transfer operator

$$
H_{p}\left(q^{-1}\right)=\frac{q^{-d} B\left(q^{-1}\right)}{A\left(q^{-1}\right)}
$$

where

$$
\begin{aligned}
B\left(q^{-1}\right) & =b_{1} q^{-1}+\cdots+b_{n_{B}} q^{-n_{B}} \\
& =q^{-1} B^{*}\left(q^{-1}\right) \\
A\left(q^{-1}\right) & =1+a_{1} q^{-1}+\cdots+a_{n_{A}} q^{-n_{A}} \\
& =1+q^{-1} A^{*}\left(q^{-1}\right) .
\end{aligned}
$$

The plant is operated in closed loop with an R-S-T (two-degree of freedom) digital controller (without lack of generality) [11]. The output of the plant operating in closed-loop is given by (see Fig. 1)

$$
\begin{aligned}
y(t+1) & =-A^{*} y(t)+B^{*} u(t-d)+C e(t+1) \\
& =-A^{*} y(t)+B^{*} q^{-d}\left[-\frac{R}{S} y(t)+\frac{T}{S} r(t)\right]+C e(t+1)
\end{aligned}
$$

where $r(t)$ is a reference signal, $u(t)$ is the plant input, $e(t)$ is a zero mean Gaussian white noise sequence, and $y(t)$ is the plant output

$$
\begin{aligned}
C\left(q^{-1}\right) & =1+c_{1} q^{-1}+\cdots+c_{n_{C}} q^{-n_{C}} \\
& =1+q^{-1} C^{*}\left(q^{-1}\right)
\end{aligned}
$$

(to simplify the notation the argument $q^{-1}$ is in many cases dropped out).

Then one can construct an asymptotically optimal predictor for the output of the closed-loop system. The predicted output will be denoted $\hat{y}(t+1)$ and the prediction error will be denoted by

$$
\varepsilon_{C L}(t+1)=y(t+1)-\hat{y}(t+1) .
$$

Lemma 1: Under the hypotheses (1), (5), and

- $d, A\left(q^{-1}\right), B\left(q^{-1}\right), C\left(q^{-1}\right)$, and the R-S-T controller are perfectly known;

- $C\left(z^{-1}\right)$ and $S\left(z^{-1}\right)$ are asymptotically stable polynomials; the asymptotically optimal one step ahead predictor for the output of the closed-loop system given by (5) which assures

$$
\begin{aligned}
\varepsilon_{C L}(t+1)= & e(t+1)+\mathcal{O}\left(t, \varepsilon_{C L}(0)-e(0), \cdots,\right. \\
& \left.\varepsilon_{C L}\left(n_{C}-1\right)-e\left(n_{C}-1\right)\right)
\end{aligned}
$$

where

$$
\lim _{t \rightarrow \infty} \mathcal{O}\left(t, \varepsilon_{C L}(0)-e(0), \cdots, \varepsilon_{C L}\left(n_{C}-1\right)-e\left(n_{C}-1\right)\right)=0
$$

is given by

$$
\hat{y}(t+1)=-\hat{A}^{*} \hat{y}(t)+\hat{B}^{*} \hat{u}(t-d)+\hat{H}^{*} \frac{\varepsilon_{C L}(t)}{S}
$$

where

$$
\hat{A}^{*}=A^{*} \quad \hat{B}^{*}=B^{*}
$$

and

$$
\begin{aligned}
\hat{H}^{*} & =C^{*} S-A^{*} S-B^{*} q^{-d} R \\
& =h_{1}+h_{2} q^{-1}+\cdots+h_{n_{H}} q^{-n_{H}+1} \\
n_{H} & =\max \left(n_{C}+n_{S}, n_{A}+n_{S}, n_{B}+d+n_{R}\right)-1 \\
\hat{u}(t) & =-\frac{R}{S} \hat{y}(t)+\frac{T}{S} r(t) .
\end{aligned}
$$

Proof: Subtracting (10) from (5) and taking into account (11) and (12) one obtains

$$
\begin{aligned}
\varepsilon_{C L}(t+1)= & -A^{*} \varepsilon_{C L}(t)-\frac{B^{*} q^{-d} R}{S} \varepsilon_{C L}(t) \\
& -\frac{\hat{H}^{*}}{S} \varepsilon_{C L}(t)+C e(t+1) \\
= & -C^{*} \varepsilon_{C L}(t)+C e(t+1)
\end{aligned}
$$

which leads to

$$
C\left[\varepsilon_{C L}(t+1)-e(t+1)\right]=0
$$

which implies (9) since $C\left(z^{-1}\right)$ has all its roots inside the unit circle.

The identification algorithm will try to recursively estimate the unknown parameters of $\hat{A}^{*}, \hat{B}^{*}$, and $\hat{H}^{*}$ using an adjustable predictor derived from (10). The polynomials $A^{*}, B^{*}$, and $H^{*}$ will be replaced by their estimates $\hat{A}^{*}(t), \hat{B}^{*}(t)$, and $\hat{H}^{*}(t)$. 


\section{A. The $X$-CLOE Algorithm}

The a priori output of the adjustable predictor is given by

$$
\begin{aligned}
\hat{y}^{\circ}(t+1) & =-\hat{A}^{*}(t) \hat{y}(t)+\hat{B}^{*}(t) \hat{u}(t-d)+\hat{H}^{*}(t) \varepsilon_{C L f}(t) \\
& =\hat{\theta}_{e}^{T}(t) \phi_{e}(t)
\end{aligned}
$$

where

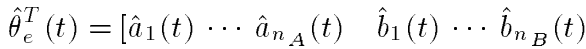

$$
\begin{aligned}
& \left.\hat{h}_{1}(t) \cdots \hat{h}_{n_{H}}(t)\right] \\
& =\left[\begin{array}{lll}
\hat{\theta}^{T}(t) & \hat{h}_{1}(t) \cdots \hat{h}_{n_{H}}(t)
\end{array}\right] \\
& \phi_{e}^{T}(t)=\left[-\hat{y}(t) \cdots-\hat{y}\left(t-n_{A}+1\right)\right. \\
& \hat{u}(t-d) \cdots \hat{u}\left(t-n_{B}+1-d\right) \\
& \left.\varepsilon_{C L f}(t) \cdots \varepsilon_{C L f}\left(t-n_{H}+1\right)\right] \\
& =\left[\phi^{T}(t) \quad \varepsilon_{C L f}(t) \cdots \varepsilon_{C L f}\left(t-n_{H}+1\right)\right] \\
& \hat{H}^{*}(t)=h_{1}(t)+h_{2}(t) q^{-1}+\cdots+h_{n_{H}}(t) q^{n_{H}-1} \\
& \varepsilon_{C L f}(t)=\frac{1}{S} \varepsilon_{C L}(t) \\
& S\left(q^{-1}\right)=1+s_{1} q^{-1}+\cdots s_{n_{S}} q^{-n_{S}}
\end{aligned}
$$

and $\hat{y}(t)$ is the a posteriori output of the adjustable predictor given by

$$
\begin{aligned}
\hat{y}(t+1)= & -\hat{A}^{*}(t+1) \hat{y}(t)+\hat{B}^{*}(t+1) \hat{u}(t-d) \\
& +\hat{H}^{*}(t+1) \varepsilon_{C L f}(t) \\
= & \hat{\theta}_{e}^{T}(t+1) \phi_{e}(t) .
\end{aligned}
$$

The $a$ priori prediction error and the a posteriori prediction error are given by

$$
\begin{aligned}
& \varepsilon_{C L}^{\circ}(t+1)=y(t+1)-\hat{y}^{\circ}(t+1) \\
& \varepsilon_{C L}(t+1)=y(t+1)-\hat{y}(t+1) .
\end{aligned}
$$

Remark: The same equations are obtained when the excitation signal $r_{u}$ is added to the output of the controller. Only the term $(T / S) r(t)$ in (5) and (13) is replaced by $r_{u}(t)$.

The recursive parameter estimation algorithm is as follows:

$$
\begin{aligned}
\varepsilon_{C L}^{\circ}(t+1) & =y(t+1)-\hat{y}^{\circ}(t+1) \\
\varepsilon_{C L}(t+1) & =\frac{\varepsilon_{C L}^{\circ}(t+1)}{1+\phi_{e}^{T}(t) F(t) \phi_{e}(t)} \\
\hat{\theta}_{e}(t+1) & =\hat{\theta}_{e}(t)+F(t) \phi_{e}(t) \varepsilon_{C L}(t+1) \\
F(t+1) & =\frac{1}{\lambda_{1}(t)}\left[F(t)-\frac{F(t) \phi_{e}(t) \phi_{e}^{T}(t) F(t)}{\frac{\lambda_{1}(t)}{\lambda_{2}(t)}+\phi_{e}^{T}(t) F(t) \phi_{e}(t)}\right] .
\end{aligned}
$$

Equation (26) is obtained by subtracting (24) from (23) and using (16), (22), and (27), observing that

$$
\begin{aligned}
\varepsilon_{C L}^{\circ}(t+1)-\varepsilon_{C L}(t+1) & =\left[\hat{\theta}_{e}(t+1)-\hat{\theta}_{e}(t)\right]^{T} \phi_{e}(t) \\
& =\phi_{e}^{T}(t) F(t) \phi_{e}(t) \varepsilon_{C L}(t+1) .
\end{aligned}
$$

\section{Stability AnALYsis}

The results of the stability analysis are presented in the following theorem.

Theorem 1: Under the following assumptions:

A1) the open-loop system is in the model set and the closed-loop system is stable;

A2) the controller is known and asymptotically stable;

A3) the external excitation $r(t)$ (or $\left.r_{u}(t)\right)$ is bounded and $\| e(t+$ 1) $\equiv 0 \|$ the recursive algorithm given by (25)-(28) with $0<\lambda_{1}(t) \leq 1$ and $0 \leq \lambda_{2}(t)<2$ assures

$$
\begin{aligned}
& \text { a) } \lim _{t \rightarrow \infty} \varepsilon_{C L}(t+1)=0 \\
& \text { b) } \lim _{t \rightarrow \infty} \varepsilon_{C L}^{\circ}(t+1)=0 \\
& \text { c) }\left\|\phi_{e}(t)\right\|<c \quad 0<c<\infty \quad \forall t
\end{aligned}
$$

for all initial conditions $\hat{\theta}_{e}(0), \varepsilon_{C L}^{\circ}(0), \phi_{e}(0)$.

Proof: Observe that adding and subtracting the term $\pm\left(A^{*}+\right.$ $\left.\left(B^{*} q^{-d} R / S\right)\right) \hat{y}(t)$ in (5) for $e(t+1) \equiv 0$, allows one to rewrite it as

$$
\begin{aligned}
y(t+1) & =-A^{*} \hat{y}(t)+B^{*} \hat{u}(t-d)-\left[A^{*} S+B^{*} q^{-d} R\right] \frac{\varepsilon_{C L}(t)}{S} \\
& =\theta_{e}^{T} \phi_{e}(t)
\end{aligned}
$$

where

$$
\begin{aligned}
& \theta_{e}^{T}(t)=\left[\begin{array}{llll}
a_{1} \cdots a_{n_{A}} & b_{1} \cdots b_{n_{B}} & h_{1} \cdots h_{n_{H}}
\end{array}\right] \\
& =\left[\begin{array}{lll}
\theta^{T} & h_{1} \cdots h_{n_{H}}
\end{array}\right] \\
& H_{0}^{*}\left(q^{-1}\right)=-\left[A^{*} S+B^{*} q^{-d} R\right] \\
& =h_{1}+h_{2} q^{-1}+\cdots+h_{n_{H}} q^{-n_{H}+1} \text {. }
\end{aligned}
$$

Subtracting (22) from (32) one gets

$$
\varepsilon_{C L}(t+1)=\left[\theta_{e}-\hat{\theta}_{e}(t+1)\right]^{T} \phi_{e}(t) .
$$

The form of (35) for the a posteriori error and (27) and (28) of the parameter adaptation algorithm allows one to use the results of [12] and (29) follows immediately. It remains to show that (30) and (31) hold.

To prove (30) one has to show that $\phi_{e}(t)$ is bounded. The components of $\phi_{e}(t)$ are $\varepsilon_{C L f}(t-i), \hat{y}(t-i)$, and $\hat{u}(t-d-i)$ where $i=0,1,2, \cdots$. Since $\varepsilon_{C L}(t)$ is bounded it results that $\varepsilon_{C L f}(t)$ is bounded [by Assumption A2)]. Assumptions A1) and A3) lead to the boundedness of $y(t)$, then from $\hat{y}(t)=y(t)-\varepsilon_{C L}(t)$ it can be concluded that $\hat{y}(t)$ is also bounded. Taking into account (13) and Assumptions A2) and A3) it results that $\hat{u}(t)$ is bounded. Therefore $\phi_{e}(t)$ is bounded.

Remark: The closed-loop output error algorithm proposed in [5], which uses an adjustable predictor of the form of (22) but without the term $\hat{H}^{*}(t+1) \varepsilon_{C L f}(t)$, requires a strictly positive real condition upon $S / P$ as a sufficient condition for global stability. The present algorithm removes this condition but the number of parameters to be estimated is increased.

\section{Convergence Analysis}

The objective is to show that in the presence of noise one obtains asymptotically an optimal predictor for the closed-loop output as well as an unbiased parameter estimation under richness conditions. We will use for this analysis a martingale-type analysis [8], [13] (the use of the averaging method (ODE) [1] leads to the same convergence condition).

In the presence of noise the plant model output [using (32)] can be expressed as

$$
\begin{aligned}
y(t+1)= & -A^{*} \hat{y}(t)+B^{*} \hat{u}(t-d)-\left[A^{*} S+B^{*} q^{-d} R\right] \varepsilon_{C L f}(t) \\
& +C e(t+1) \\
= & \theta^{T} \phi(t)-\left[A^{*} S+B^{*} q^{-d} R\right] \varepsilon_{C L f}(t)+C e(t+1) .
\end{aligned}
$$

Subtracting (22) from (36) the a posteriori prediction error equation becomes

$$
\begin{aligned}
\varepsilon_{C L}(t+1)= & {[\theta-\hat{\theta}(t+1)]^{T} \phi(t)+\left[-A^{*} S-B^{*} q^{-d} R\right.} \\
& \left.-\hat{H}^{*}(t+1)\right] \varepsilon_{C L f}(t)+C e(t+1) .
\end{aligned}
$$


Adding and subtracting the term $\pm C^{*} \varepsilon_{C L}(t),(37)$ can be rewritten as

$$
\varepsilon_{C L}(t+1)=-C^{*} \varepsilon_{C L}(t)+\left[\theta_{e}-\hat{\theta}_{e}(t+1)\right]^{T} \phi_{e}(t)+C e(t+1)
$$

where

$$
\begin{aligned}
\theta_{e}^{T} & =\left[a_{1} \cdots a_{n_{A}}, b_{1} \cdots b_{n_{B}}, h_{1} \cdots h_{n_{H}}\right] \\
& =\left[\theta^{T}, h_{1}, \cdots, h_{n_{H}}\right] \\
H^{*}\left(q^{-1}\right) & =C^{*} S-A^{*} S-B^{*} q^{-d} R \\
& =h_{1}+h_{2} q^{-1}+\cdots+h_{n_{H}} q^{-n_{H}+1} .
\end{aligned}
$$

From (38) one obtains

$$
\varepsilon_{C L}(t+1)=\frac{1}{C}\left[\theta_{e}-\hat{\theta}_{e}(t+1)\right]^{T} \phi_{e}(t)+e(t+1) .
$$

For convergence analysis the sequence $\{e(t)\}$ will be taken as a martingale difference sequence defined on a probability space $(\Omega, A, P)$ adapted to the sequence of increasing $\sigma$-algebra generated by the observations up to and including time $t$. The sequence $\{e(t)\}$ is assumed to satisfy the following properties:

$$
\begin{aligned}
E\left\{e(t+1) \mid \mathcal{F}_{t}\right\} & =0 \\
E\left\{e^{2}(t+1) \mid \mathcal{F}_{t}\right\} & =\sigma^{2} \\
\lim _{N \rightarrow \infty} \sup \frac{1}{N} \sum_{t=1}^{N} e^{2}(t) & <\infty .
\end{aligned}
$$

The usual model given by a sequence of equally distributed normal random variables $(0,1)$ satisfies the above properties. One has the following result.

Theorem 2: Consider the parameter adaptation algorithm given by (25)-(28) with $\lambda_{1}(t)=1$ and $\lambda_{2}(t)=1$. Assume that $\{e(t+1)\}$ is a martingale sequence satisfying the properties (42)-(44). If the transfer function

$$
H^{\prime}\left(z^{-1}\right)=\frac{1}{C\left(z^{-1}\right)}-\frac{1}{2}
$$

is strictly positive real, then

$$
\begin{aligned}
& \lim _{N \rightarrow \infty} \frac{1}{N} \sum_{t=1}^{N}\left[\varepsilon_{C L}(t)-e(t)\right]^{2}=0 \quad \text { a.s. } \\
& \lim _{N \rightarrow \infty} \frac{1}{N} \sum_{t=1}^{N} \varepsilon_{C L}^{2}(t)=\lim _{N \rightarrow \infty} \frac{1}{N} \sum_{t=1}^{N} e^{2}(t) \quad \text { a.s. } \\
& \lim _{N \rightarrow \infty} \frac{1}{N} \sum_{t=1}^{N}\left[\left[\theta_{e}-\hat{\theta}_{e}(t)\right]^{T} \phi_{e}(t-1)\right]^{2}=0 \quad \text { a.s. } \\
& \lim _{N \rightarrow \infty} \frac{1}{N} \sum_{t=1}^{N}\left[\theta_{e}-\hat{\theta}_{e}(t)\right]^{T} \frac{F^{-1}(t)}{t}\left[\theta_{e}-\hat{\theta}_{e}(t)\right]=0 \quad \text { a.s. }
\end{aligned}
$$

If in addition $\lim _{t \rightarrow \infty}(1 / t) F^{-1}(t)>0$ (a.s.) then $\lim _{t \rightarrow \infty} \hat{\theta}_{e}(t)=$ $\theta_{e}$ (a.s.).

Proof: The proof of Theorem 2 is a straightforward application of [8, Th. 4.2] taking into account the form of (25), (26), and (41), provided that one can prove first that $\lim _{N \rightarrow \infty}(1 / N) \sum_{t=1}^{N} \phi_{e}^{T}(t) \phi_{e}(t)<\infty$. This condition can be immediately verified using [8, Lemma 4.1], which is a generalization of a result from [6]. The components of the vector $\phi_{e}$ satisfy the condition

$\frac{1}{N} \sum_{t=1}^{N}\left|\phi_{e i}(t)\right|^{2} \leq K_{1}+\frac{K_{2}}{N} \sum_{t=1}^{N} \varepsilon_{C L}^{2}(t-i), \quad 0<K_{1}, K_{2}<\infty$

by the way they are obtained, and taking into account that $y(t)$ and $r(t)$ are bounded. Therefore, it is enough to show that

$$
\lim _{t \rightarrow \infty} \frac{1}{N} \sum_{t=1}^{N} \varepsilon_{C L}^{2}(t)<\infty
$$

and this according to [8, Lemma 4.1] will hold if

$$
\lim _{t \rightarrow \infty} \frac{1}{N} \sum_{t=1}^{N}\left[\hat{\theta}_{e}^{T}(t) \phi_{e}(t)+\varepsilon_{C L}^{\circ}(t+1)\right]^{2}<\infty .
$$

But

$$
\hat{\theta}_{e}^{T}(t) \phi_{e}(t)+\varepsilon_{C L}^{\circ}(t+1)=y(t+1)
$$

which is bounded, therefore (46) is true. The results in [13] can also be used for an alternative proof.

\section{CONCLUSION}

A recursive algorithm for ARMAX plant model identification in closed-loop operation has been presented. The stability analysis in a deterministic environment has shown the global asymptotic stability of the algorithm without requiring a positive real condition. The convergence analysis in a stochastic environment has shown that asymptotic unbiased parameter estimates can be obtained under the same condition on the noise model as in open-loop identification. However, compared to the open-loop case a larger number of parameters has to be estimated.

\section{REFERENCES}

[1] L. Ljung, System Identification-Theory for the User. Englewood Cliffs, NJ: Prentice-Hall, 1987.

[2] T. Söderström and P. Stoica, System Identification. U.K.: PrenticeHall, 1989.

[3] I. Gustavsson, L. Ljung, and T. Söderström, "Identification of processes in closed loop-Identifiability and accuracy aspects," Automatica, vol. 13, pp. 59-75, Apr. 1977.

[4] M. Gevers, "Toward a joint design of identification and control?," in Essays on Control, Perspectives in the Theory and its Applications, $\mathrm{H}$ L. Trentelman and J. C. Willems, Eds. Boston, MA: Birkhäuser, 1993.

[5] I. D. Landau and A. Karimi, "An output error recursive algorithm for unbiased identification in closed loop," Automatica, vol. 33, no. 5, pp. 933-938, May 1997.

[6] V. Solo, "The convergence of AML," IEEE Trans. Automat. Contr., vol. 24, pp. 958-963, 1979.

[7] L. Dugard and I. D. Landau, "Recursive output error identification algorithms," Automatica, vol. 16, pp. 443-462, 1980.

[8] I. D. Landau, "Near supermartingales for convergence analysis of recursive identification and adaptive control schemes," Int. J. Contr., vol. 35, no. 2, pp. 197-226, 1982.

[9] I. D. Landau and A. Karimi, "A recursive algorithm for armax model identification in closed loop," Lab. d'Automatique de Grenoble, ENSIEG, 38402 St. Martin d'Heres, Tech. Rep., 1996.

[10] A. Karimi and I. D. Landau, "Comparison of the closed loop identification methods in terms of the bias distribution," Syst. Contr. Lett., vol. 34, pp. 159-167, 1998.

[11] I. D. Landau, System Identification and Control Design. Englewood Cliffs, NJ: Prentice-Hall, 1990.

[12] _ _ "An extension of a stability theorem applicable to adaptive control," IEEE Trans. Automat. Contr., vol. AC-25, pp. 814-817, 1980.

[13] G. C. Goodwin and K. S. Sin, Adaptive Filtering Prediction and Control. Englewood Cliffs, NJ: Prentice-Hall, 1984. 